\title{
A $10 \mathrm{GHz}$ Quasi-Optical Grid Amplifier Using Integrated HBT Differential Pairs
}

\author{
Moonil Kim a, E. A. Sovero b, W. J. Ho b, J. B. Hacker a,David B. Rutledge a, \\ James J. Rosenberg $c$, and R. Peter Smith $d$ \\ aDivision of Engeineering and Applied Science, California Institute of Technology, Pasadena CA 91125 \\ bscience Center, Rockwell International Corporation, P. O. Box 1085, 1049 Camino Dos Rios, Thousand Oaks, CA 91358 \\ cGermanium Power Devices Corporation, P. O. Box 3065 SVS, Andover, MA 01810 \\ dCenter for Space Microelectronic Technology, Jet Propulsion Laboratory, 4800 Oak Grove Drive, Pasadena, CA 91109
}

We report the fabrication and testing of a $10 \mathrm{GHz}$ grid amplifier utilizing sixteen GaAs chips each containing an HBT differential pair plus integral bias/feedback resistors. The overall amplifier consists of a $4 \times 4$ array of unit cells on an RT Duroid ${ }^{\mathrm{TM}}$ board having a relative permittivity of 2.2 . Each unit cell consists of an emitter-coupled differential pair at the center, an input antenna which extends horizontally in both directions from the two base leads, an output antenna which extends vertically in both directions from the two collector leads, and high inductance bias lines. In operation, the active grid array is placed between a pair of crossed polarizers. The horizontally polarized input wave passes through the input polarizer and couples to the input leads. An amplified current then flows on the vertical leads, which radiate a vertically polarized amplified signal through the output polarizer. The polarizers serve dual functions, providing both input-output isolation as well as independent impedance matching for the input and output ports. The grid thus functions essentially as a free-space beam amplifier. Calculations indicate that output powers of several watts per square centimeter of grid area should be attainable with optimized structures.

Quasi-optical grid devices including oscillators, multipliers, mixers, and beam steerers have already been demonstrated $[1,2,3,4]$. Motivation for studying quasi-optical devices is two-fold: free-space power combining is more efficient at high frequencies than power combining in guided wave structures, and transmitting and receiving systems based on monolithic implementations of quasi-optical elements have the potential for being smaller, lighter, and substantially less costly than conventional phased-array systems.

The AlGaAs/GaAs HBT material was grown by MBE.

$\begin{array}{lllll}\text { LAYER } & \text { THCKNESS }(\mu \mathrm{m}) & \text { TYPE } & \text { DOPING }\left(\mathrm{cm}^{-3}\right) & \text { AIAs FRACTION } \\ \text { Cap } & 0.16 & \mathrm{n}^{+} & 5 \times 10^{18} & 0 \\ \text { Emitter } & 0.1 & \mathrm{n}^{-} & 0.5-1.5 \times 10^{18} & 0-.25-0 \\ \text { Base } & 0.07 & \mathrm{p}^{+} & 0.5-1.0 \times 10^{20} & 0 \\ \text { Collector } & 0.7 & \mathrm{n} & 3-6 \times 10^{16} & 0 \\ \text { Subcollector } & 0.6 & \mathrm{n}^{+} & 6 \times 10^{18} & 0\end{array}$

The devices are implant isolated and have an active emitter area of 40 square microns. The transistors exhibit $f_{T}=65 \mathrm{GHz}$, and $f_{\max }=90 \mathrm{GHz}$. Each chip contains a differential pair with $1.7 \mathrm{k} \Omega$ collector-base feedback resistors and a $250 \Omega$ emitter bias resistor which also serves to supress common mode gain.

The amplifier exhibits a peak gain of $12 \mathrm{~dB}$ at $9.9 \mathrm{GHz}$, with a $3 \mathrm{~dB}$ bandwidth which extends from 9.55 $\mathrm{GHz}$ to $10.3 \mathrm{GHz}$. The peak gain and bandwidth are sensitive to polarizer position, indicating that the polarizers provide good matching to free space (as a reference point, the transistors have 18dB unilateral gain at $10 \mathrm{GHz}$ ). Output power is linear with input power, indicating that the grid operates as an amplifier rather than as an injection-locked oscillator. Gain saturation can be observed at low dc bias and high input rf levels.

This amplifier represents a significant advance over the previously reported grid amplifier [5] beyond its higher operating frequency, larger gain, and wider bandwidth. Where the previous grid required both front and backside wiring on the substrate (which would be cumbersome in a monolithic implementation), this amplifier utilizes frontside wiring only. Where the previous amplifier utilized packaged discrete MESFETs, in this amplifier the contents of each unit cell are monolithically integrated with the exception of the metal lines which make up the antenna array. This amplifier therefore represents a substantial step toward monolithic integration of an entire grid, which will be required for higher frequency operation and low cost.

\section{References}

[1] 2.B. Popovic, M. Kim, D. B. Rutledge, "Grid oscillators," Int. J. Infrared and Millimeter Waves, vol 9. no. 7, pp 647-654, 1988.

[2] R. J. Hwu, L. P. Sadwick, N. C. Luhmann, D. B. Rutledge, M. Sokolich, B. Hanoock, "Theoretical and Experimental Imvestigation of Watt-Level Wafer Scale integration of Microwave and Millimeter-Wave GaAs and AlGaAs Frequency Multipliers," 47th Device Research Conference, Boston, MA, June 1989.

[3] J. B. Hacker, R. M. Weikle II, M. Kim, M. P. Delisio, D. B. Ruttedge, "A 100-Element Planar Schotthy Diode Grid Mixer." IEEE Trans. Microwave Theory Tech., vol. 40, no. 3, March 1992.

[4] M. Kim, R. M. Weikle II, J. B. Hacker, D. B. Rutledge, "Beam diffraction by a Planar Grid Structure at 93 GHz," IEEE Antenna and Propagation Society Symposium, London. Ontario, June 1991.

[5] M. Kim, J. J. Rosenberg, R. P. Smith, R. M. Weikle, J. B. Hacker, M. P. DeLisio, D. B. Rutledge, "A Grid Amplifier," IEEE Microwave and Guided Wave Letters, vol. 13, ro. 11. pp 322-324, 1991. 


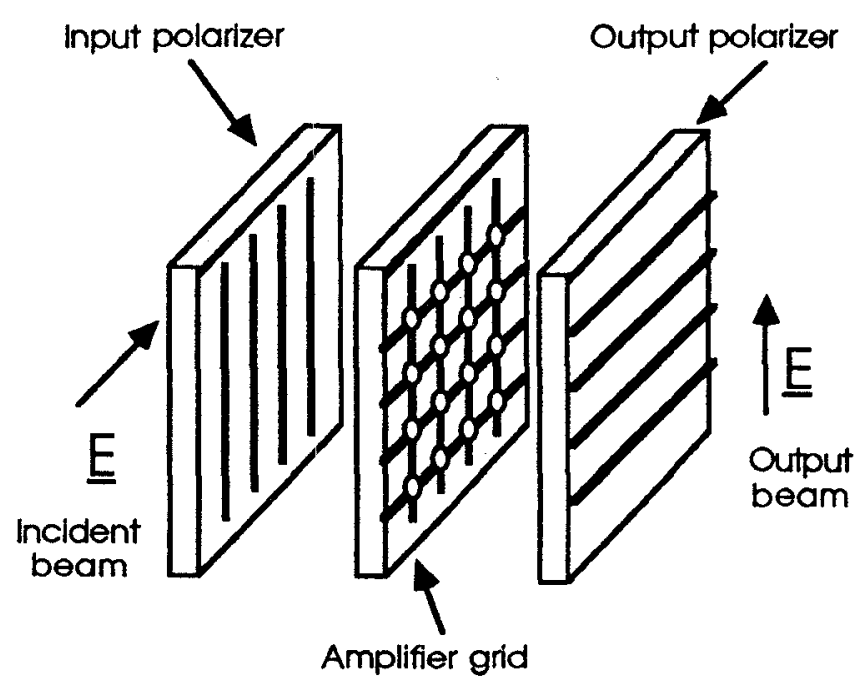

Figure 1: Perspective view of Grid Amplifier. Horizontally polarized input wave is incident from the left: vertically polarized output wave is radiated to the right. The polarizers consist of parallel copper lines on Durold boards

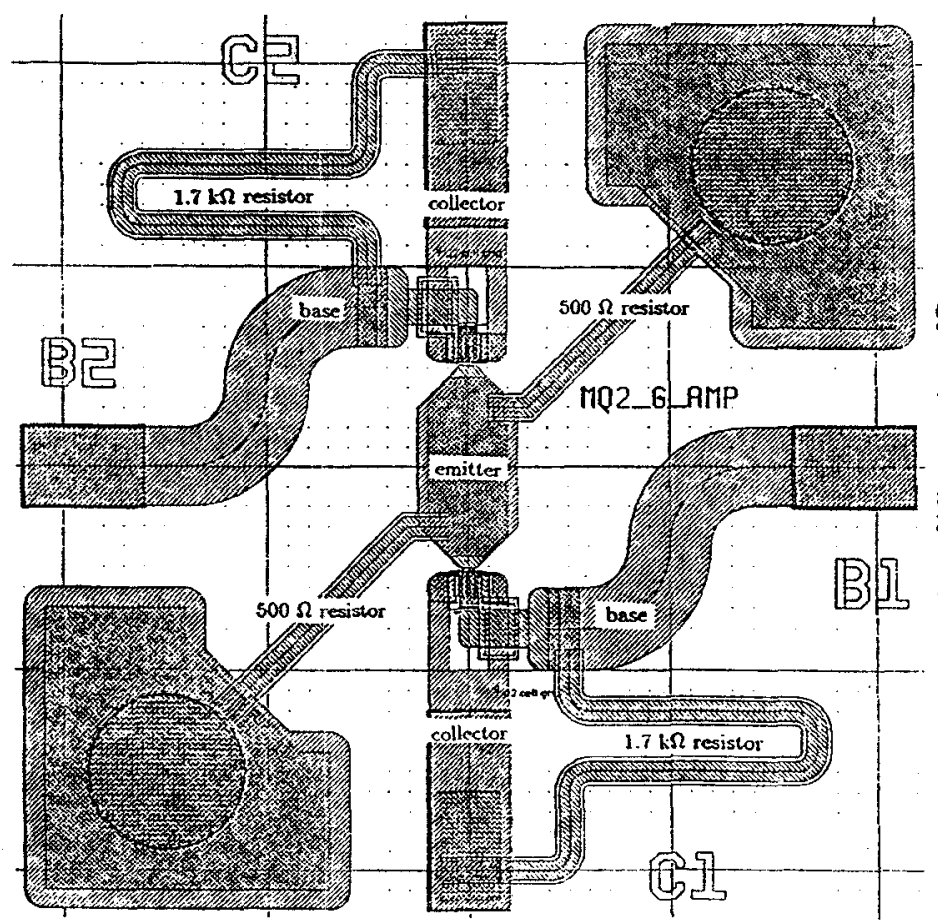

Figure 3: Plan view of the Rockwell differentia pair HBT chip. The $250 \Omega$ emitter bias resistor is implemented as a parallel combination of two $500 \Omega$ resistors for symmetry reasons.

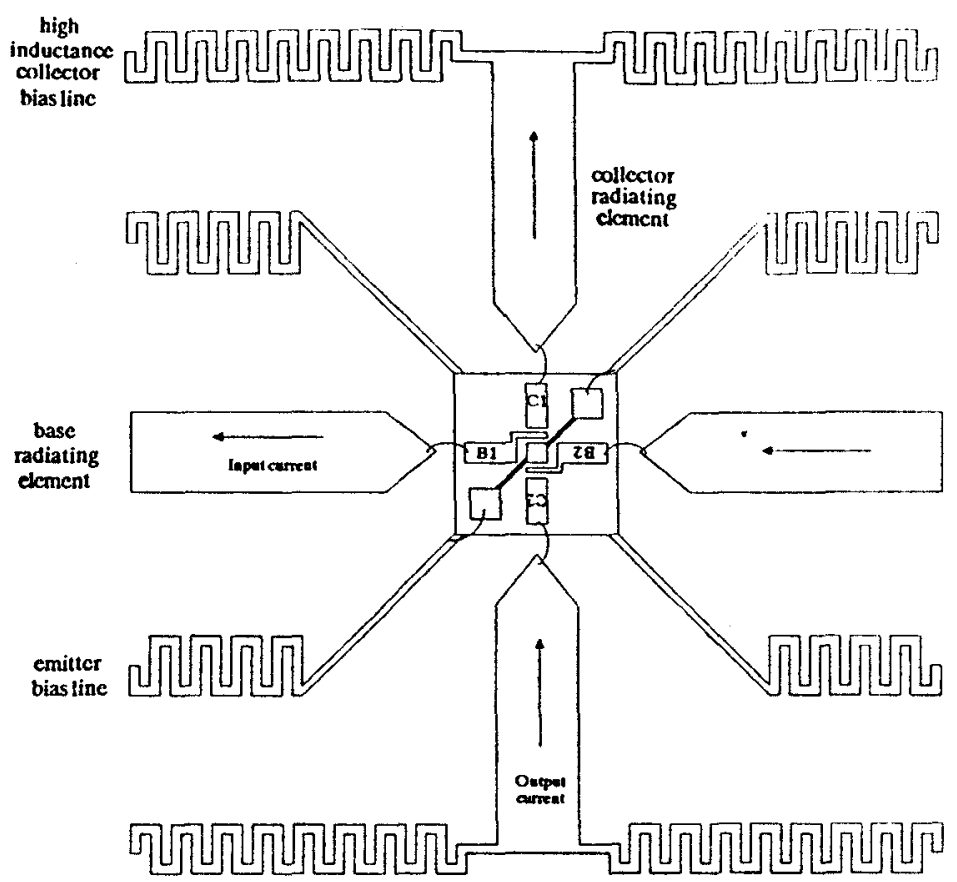

Figure 2: Plan view of unit cell. These cells are perlodically repeated in a two dimensional array. The HBT differential pair chips are bonded to the centers of the antenna pattems which are etched into a copper clad Durold board. Note that the emitter blas lines are joined beneath the chip.

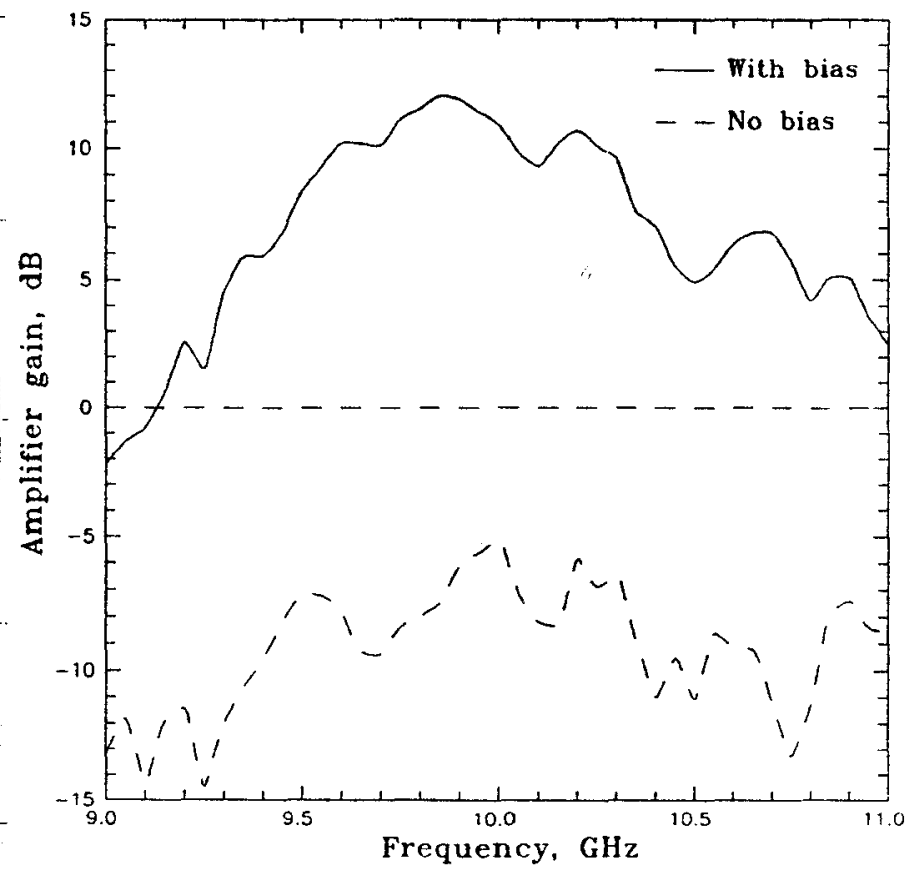

Figure 4: Gain versus frequency plot for Grid Amplifier. Output power is proportional to input power for the entire dynamic range of our measurement apparatus ( $>30 \mathrm{~dB}$ ) with $5 \mathrm{~V}$ bias supplied to the grid. At $2.5 \mathrm{~V}$ bias, grid can be saturated ot high if input power. 